\title{
Knowledge Portals
}

\author{
Dr. Steffen Staab \\ Senior Researcher and Lecturer \\ Knowledge Management Group \\ Applied Computer Science Institute (AIFB) \\ University of Karlsruhe, Germany \\ ssteaifl. unikarlsruhe. de \\ www.aifb.unikarlsruhe.de/ sst
}

Knowledge portals provide views onto domainspecific information on the World Wide Web, thus facilitating their users to find relevant, domainspecific information. The construction of intelligent access and the provisioning of information to knowledge portals, however, remained an ad hoc task requiring extensive manual editing and maintenance by the knowledge portal providers. In order to diminish these efforts we use ontologies as a conceptual backbone for providing, accessing and structuring information in a comprehensive approach for building and maintaining knowledge portals. We have built several experimental and one commercial knowledge portal for knowledge management tasks such as skill management and corporate history analysis that show how our approach is used in practice. This practice, however, has exhibited a number bottlenecks, many of which could be avoided or at least diminished by Human Language Technology. We have used HLT in order to reduce the costs of ontology engineering and in order to narrow the gap between finding knowledge in texts and providing it to the portal. 